\title{
Hyperparasites Influence Population Structure of the Chestnut Blight Pathogen, Cryphonectria parasitica
}

\author{
Joshua C. Springer, Anita L. Davelos Baines, Dennis W. Fulbright, Matthew T. Chansler, and Andrew M. Jarosz
}

First, fourth, and fifth authors: Department of Plant Biology, Michigan State University, East Lansing 48824; first and fifth authors: Program in Ecology, Evolutionary Biology, and Behavior, Michigan State University, East Lansing 48824; second author: Biology Department, University of Wisconsin-La Crosse, La Crosse 54601; and third and fifth authors: Department of Plant, Soil and Microbial Sciences, Michigan State University, East Lansing 48824.

Accepted for publication 26 June 2013.

\begin{abstract}
Springer, J. C., Davelos Baines, A. L., Fulbright, D. W., Chansler, M. T., Jarosz, A. M. 2013. Hyperparasites influence population structure of the chestnut blight pathogen, Cryphonectria parasitica. Phytopathology 103:1280-1286.

Vegetative compatibility (VC) is commonly used to characterize structure and diversity in fungal populations. In the chestnut blight fungus, Cryphonectria parasitica, high VC diversity is hypothesized to be responsible for the failure of hyperparasitic mycoviruses to spread through pathogen populations in North America. To test this hypothesis, we assessed VC diversity at three recovering sites in Michigan where mycoviruses had invaded and compared them with four epidemic population sites where mycoviruses were absent. VC diversity was assessed

between the sample dates. Sites where mycoviruses were present had unique VC structures with the exception of the recovering population site at County Line where the main VC group was also detected at two epidemic sites. With one exception, epidemic sites contained more VC groups and displayed higher population level diversity than recovering sites. Mating-type analyses of blight populations revealed that two of three recovering populations were significantly skewed for MAT2 suggesting asexual reproduction, while epidemic sites with a long history of blight infection had ratios near 50:50 suggesting sexual reproduction. We propose that selection in the largely asexual $C$. parasitica populations at two recovering sites favors the most-fit fungal genotype by mycovirus combination and results in reduced diversity relative to the sexually reproducing pathogen populations at epidemic sites.
\end{abstract} for samples collected in 1996 and 2009, which allowed us to determine how $C$. parasitica populations changed with time. Twelve VC types were found in 1996 while 29 were found in 2009; 75\% of types overlapped
Additional keywords: balancing selection, frequency-dependent selection, mating-type alleles, vegetative incompatibility.
Introduced pathogens have a long history of causing extensive destruction to their plant hosts, with trees being particularly vulnerable. For example, Dutch elm disease caused by Ophiostoma ulmi and O. novo-ulmi has changed the urban landscape in the United States and Europe by killing highly susceptible elms planted as shade trees $(9,10,45,52)$. American chestnuts (Castanea dentata L.) have suffered a similar fate in North America. Prior to the 1900 s, $C$. dentata made up nearly $25 \%$ of the canopy in eastern hardwood forests, but these natural populations were decimated when Cryphonectria parasitica Murrill (Barr.), the cause of chestnut blight, was accidentally introduced to the United States in the early twentieth century near New York City (36). Cryphonectria parasitica enters wounds on the trunk and branches of susceptible trees and causes a canker that expands in size until the branch or trunk is girdled, killing all plant material distal to the canker. Trees accumulate multiple infections during an epidemic and eventually all aboveground portions of the tree are killed. However, $C$. parasitica cannot enter the roots of infected trees and chestnuts can survive for considerable time as a live root system that periodically produces root collar sprouts. These sprouts grow for several seasons before being infected and killed by $C$. parasitica. Consequently, $C$. dentata is still a common, persistent, understory tree in several areas $(4,25,35)$.

C. parasitica was also introduced in Europe in the 1930s and the initial stages of the European blight epidemic were similar to

Corresponding author: A. M. Jarosz; E-mail address: amjarosz@msu.edu

http://dx.doi.org/10.1094/PHYTO-10-12-0273-R

(c) 2013 The American Phytopathological Society that in North America. However, European chestnuts (Castanea sativa) began recovering by the 1950s (26). Canker expansion on recovering trees was contained by wound callus tissue, which prevented girdling of the trunk and allowed infected trees to continue growing. Recovery was not due to resistance in European chestnuts (although their reduced susceptibility relative to American chestnuts may play a role), but was caused by reduced pathogen virulence induced by the presence of double-stranded RNA mycovirus hyperparasites $(24,32,39)$. Mycoviruses reduce pathogen virulence by reducing fungal growth, decreasing conidia production, and inhibiting sexual reproduction (i.e., ascospore production) and therefore can act as a biological control mechanism against the blight pathogen $(3,12,17,35)$. Over time, mycoviruses spread to many areas in Europe and are thought to be responsible for the recovery of Castanea sativa. With the exception of some isolated populations in Michigan, mycoviruses are relatively rare in North America and efforts to introduce them as biological control have been largely unsuccessful (39).

Considerable speculation on why mycoviruses have not invaded North American populations of $C$. parasitica has centered on the higher diversity of vegetative compatibility (VC) groups in North America relative to Europe (5). VC groups are a consequence of a self/non-self recognition system found in many ascomycetes that are controlled by several unlinked vegetative incompatibility (vic) or heterokaryon incompatibility (het) loci (21). The incompatibility loci operate to detect non-self hyphae when the hyphal tips of adjacent colonies fuse (anastomosis). Mismatches at any incompatibility locus trigger apoptosis (programmed cell death) causing the connection between mismatched hyphae to die (for review, see Glass and Kaneko [22]). Apoptosis prevents alien 
nuclei from invading the fungal colony and reduces the transfer of cytoplasmic elements such as mycoviruses $(3,5,28,31,32,38)$. Alleles at vic loci are thought to be under negative frequency dependent or balancing selection which maximizes the ability of individuals to detect non-self hyphae upon fusion $(22,37,40,55)$ and promotes high levels of diversity for VC groups within fungal populations (Table 1 of Nauta and Hoekstra [42]). However, theoretical work by Taylor et al. (54) and Morozov et al. (41) suggest that the vic incompatibility system may not be a complete barrier to mycovirus invasion since mismatches at one or two vic loci reduces but does not entirely prevent transmission of mycoviruses between fungal colonies $(28,31)$.

Here we address a different aspect of the interaction between the chestnut blight pathogen and its mycoviruses by comparing seven $C$. parasitica populations in Michigan to determine how invasion by mycoviruses influences the VC structure of the pathogen. Mycoviruses have been present for an extended period of time (since at least the early 1980s) at three of our study sites, County Line, Frankfort, and Roscommon $(7,15,19)$, while they are largely absent at the other four sites (15). In 1996 when this study was initiated, more than $90 \%$ of all C. parasitica samples from the three recovering sites contained mycoviruses (15). Mycoviruses have a substantial positive influence on chestnut trees at the three sites by increasing tree survivorship, growth, and reproduction resulting in a general "recovery" of these chestnut populations (hereafter recovering sites). Indeed, the population demographics at two of the recovering sites are similar to those of disease-free chestnut populations in Michigan (16). In contrast, trees at the four sites without mycoviruses have demographics similar to that found in the main range of the American chestnut where all large trees are dying back as a result of the C. parasitica epidemics (hereafter epidemic sites).

What is not known is the extent to which invasions by mycoviruses influence the population structure of $C$. parasitica. Mycoviruses would be expected to alter the population genetics of C. parasitica since they inhibit the pathogen's sexual cycle (23). The lack of a sexual cycle in mycovirus-infected individuals of C. parasitica would be expected to have multiple influences on pathogen population structure. (i) Diversity of VC groups should decrease because genetic recombination through sexual reproduction does not occur on a regular basis. (ii) Selection will operate at the whole-genome level in asexual populations of the fungal pathogen because of the lack of recombination. (iii) Dispersal of the fungal pathogen should be altered because the winddispersed sexual ascospores are not produced and the splashdispersed asexual spores (i.e., conidia) have a more limited dispersal distance.

Our objectives were to compare the VC structure of $C$. parasitica found at the three recovering populations with that of the four epidemic populations to determine how mycoviruses influence VC structure over time. Additionally, we investigated the temporal pattern at five sites to determine if the pattern of variability differed between recovering and epidemic populations from 1996 to 2009.

\section{MATERIALS AND METHODS}

Chestnut blight cankers were sampled from five blighted American chestnut populations in 1996 and seven in 2009. Thirty haphazard samples from each population were collected in 2009, while the number of samples used for comparison from 1996 was variable since we were unable to resurrect some samples from storage (J. C. Springer and M. T. Chansler, unpublished data). Three of the blighted tree populations, County Line (CL), Roscommon (RC), and Frankfort (FR), have been invaded naturally by double-stranded RNA mycoviruses and the American chestnut trees at those sites are recovering from the blight epidemic (16, A. M. Jarosz and J. C. Springer, unpublished data). However, in recent years, many trees at the Frankfort site have died due to blight infections (A. M. Jarosz and J. C. Springer, unpublished data). The four remaining sites, Stivers (ST), Missaukee diseased (MD), Missaukee healthy (MH), and Leelanau (LE), are experiencing blight epidemics that have severely debilitated the chestnut trees; mycoviruses have not been found at these sites at a level higher than $5 \%$ of cankers sampled (15, J. C. Springer, unpublished data). Population location coordinates can be found in Davelos and Jarosz (16) for all sites except RC. The RC site is located at $44^{\circ} 31^{\prime} 28^{\prime \prime} \mathrm{N},-84^{\circ} 30^{\prime} 48^{\prime \prime} \mathrm{W}$ (Figs. 1 and 2 provide general locations). Blight was first found at MH and LE in 1997; samples were obtained from these sites only in 2009. Samples from 1996 were resurrected from sterile water storage vials inoculated with mycelial plugs; $200 \mu \mathrm{l}$ of water from each vial was plated onto potato dextrose agar (PDA) petri plates (Difco, Becton, Dickinson and Company, Sparks, MD). For 2009 samples, subsamples $(2 \times$ $2 \mathrm{~mm}^{2}$ ) of each bark collection were surface sterilized in $10 \%$ sodium hypochlorite solution for $20 \mathrm{~s}$, rinsed in double distilled $\mathrm{H}_{2} \mathrm{O}$ twice for $20 \mathrm{~s}$, and clean isolates were plated onto PDA.

Since the presence of mycoviruses can obscure VC testing (D. W. Fulbright, personal observation), mycovirus-free isolates were obtained from all samples before testing for VC. Since, transmission of mycovirus to conidia is rarely $100 \%(18,51)$, mycovirus-free isolates were obtained by plating serially diluted single conidia onto PDA plates and an isolate with a fast-growing virulent phenotype was chosen. VC tests were performed on debarked chestnut stem pieces embedded in PDA. Chestnut pieces were approximately $1 \mathrm{~cm}$ long, cut longitudinally and placed in standard petri plates $\left(100 \times 15 \mathrm{~mm}^{2}\right)$ (Medegen, Gallaway, $\mathrm{TN}$ ) according to Huber (28). Isolates were allowed to grow across the chestnut stem pieces for approximately 2 weeks before compatibility reactions were scored as either compatible or incompatible using evidence of a solid mat of mycelia or barrage formation between isolates, respectively (Fig. 3). An internal control of two isolates from the same canker was included on each plate. Fresh isolates of similar age (usually less than 1 week old) were used for testing since they exhibited rapid, consistent growth on the petri plate. VC types were determined visually, since the formation of barrage lines is sufficient to determine that cultures differ for VC group.

For mating-type analysis, isolates were grown on PDA plates overlaid with sterilized cellophane. After approximately 2 weeks, fungal mats were scraped off of the cellophane and dried at room temperature overnight. Isolates were ground to a fine powder in mortars with liquid nitrogen. DNA extractions were performed with 25 to $30 \mathrm{mg}$ of powdered fungus with a Qiagen plant minikit (Qiagen Corporation, Valencia, CA) according to manufacturer directions. Primer sequences for MAT1 and MAT2 and thermocycler protocol follow previous work of Marra and Milgroom (34). GoTaq Green master mix (Promega Corporation, Madison, WI) was used according to manufacturer directions in place of separate PCR components. PCR products ( $5 \mu \mathrm{l}$ subsample) were run on a $1 \%$ agarose gel for $35 \mathrm{~min}$ at $90 \mathrm{v}$ and visualized under UV light and scored according to migration distance on the gel (34). Chi-square tests were performed on the frequencies of the two MAT alleles in each site to determine if populations diverged from the 50:50 ratio expected if the pathogen population was sexually reproducing.

Data analysis. The lack of a sexual cycle in mycovirus-infected individuals of $C$. parasitica would be expected to have multiple influences on pathogen population structure: (i) diversity of VC groups should decrease because genetic recombination does not occur on a regular basis; (ii) selection will operate at the wholegenome level in asexual populations of the fungal pathogen because of the lack of recombination; and (iii) dispersal of the fungal pathogen should be altered because the wind-dispersed sexual ascospores are not produced and the splash-dispersed asexual spores (i.e., conidia) have a more limited dispersal distance. 
To specifically address these predictions, we asked the following questions. (i) Do populations with mycovirus have lower VC diversity than populations without mycovirus? (ii) Does VC diversity decrease over time in populations with mycovirus? If yes, this result would be consistent with selection for a most fit fungus-mycovirus combination at each site, i.e., one or few VC types infected by mycoviruses that are unique and lead to chestnut recovery. (iii) Are population structures similar in populations that are spatially close? If yes, this pattern would be consistent with limited dispersal of spores.

To address the first two questions, diversity measures were calculated. Alpha diversity, used here as population level VC richness, was calculated as the total number of VC groups within a population. Strong weighting to dominant members of the community of interest is given by Simpson's index (27). Simpson's diversity index is based on the formula

$$
D_{S}=1-\left[\sum n_{i}\left(n_{i}-1\right) / N(N-1)\right]
$$

where $n_{i}$ is the number of isolates in a VC group and $N$ is the total number of isolates. A moderate weighting to rare and inter- mediate species in comparison to dominant species is provided by the Shannon index. Thus, the Shannon index is more sensitive to changes in abundance of rare groups in comparison to other diversity indices (27). The Shannon diversity index is based on the formula

$$
\left.H=-\sum x_{i} / x_{o}\right) \ln \left(x_{i} / x_{o}\right)
$$

where $x_{i}$ is the number of isolates in a VC group and $x_{o}$ is the total number of isolates. Diversity indices and standard deviations for epidemic and recovering populations were calculated using EstimateS (13).

Morisita's index of dispersion measures overlap of VC types among samples or populations (33) and can be thought of as a probability. Index values are generated using the formula

$$
\left.C_{D}=2 \sum_{i=1}^{S} x_{i} y_{i} /\left(D_{x}+D_{y}\right) X Y\right)
$$

where $x_{i}$ represents how many instances species $i$ is in the total $X$ in one population, and where $y_{i}$ represents how many instances VC group $i$ is in the total $Y$ from another population. $D_{x}$ and $D_{y}$

\section{Missaukee Diseased*}

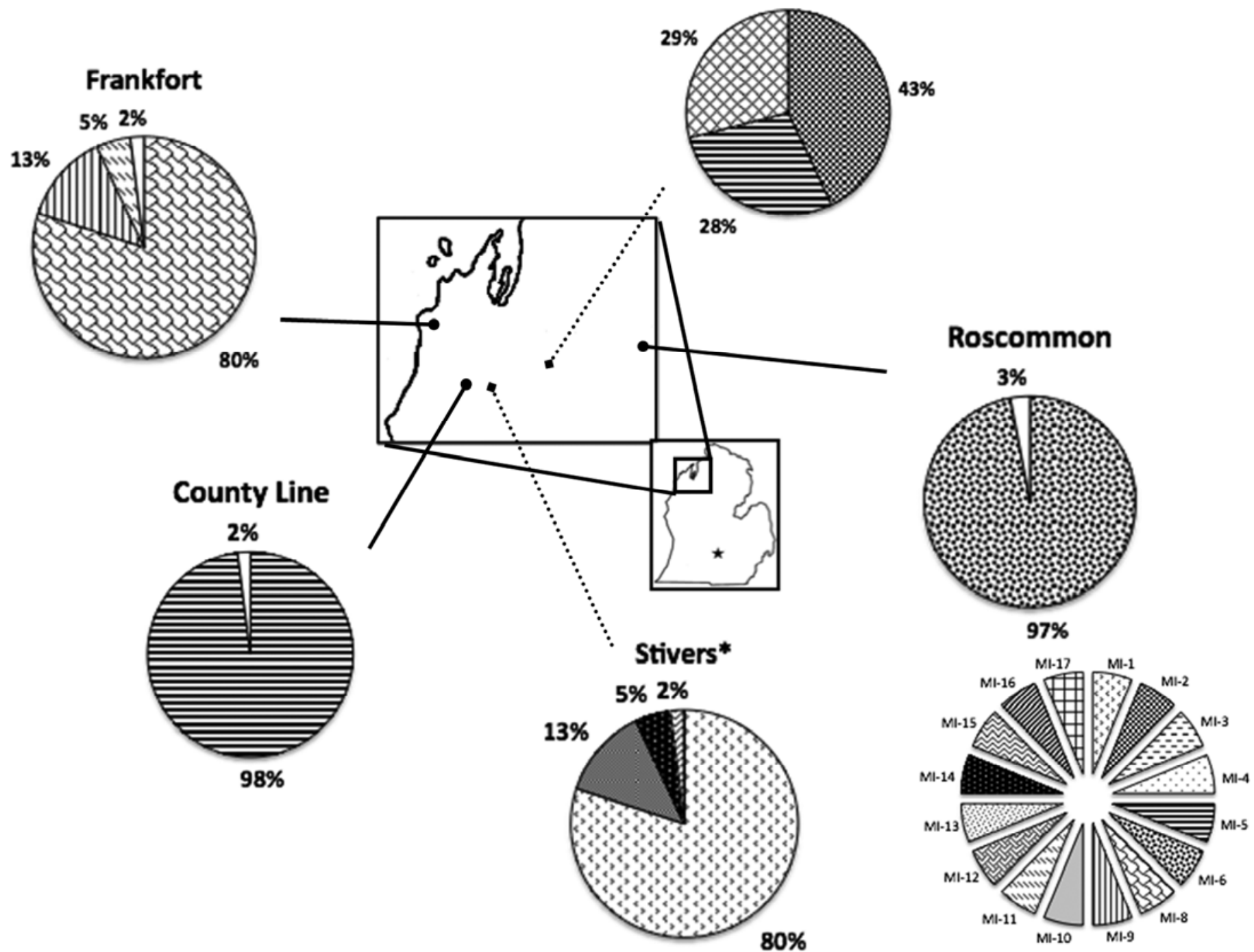

Fig. 1. Vegetative compatibility diversity at five Michigan population sites for 1996. Rare types at recovering and epidemic populations are represented by white and black slices, respectively. Recovering sites are denoted with solid circles, epidemic sites with solid squares. Asterisk indicates 1996 data for Stivers and Missaukee Diseased represent only those samples that could be resurrected from storage; see text for details. 
are replaced by Simpson's values according to population $x$ and $y$ and $S$ is the number of species unique in a population. Morisita's index values range from 0 (no similarity between populations) to 1 (total similarity). The matrices of Morisita's index values and physical distances (in kilometers) for the seven populations were compared with each other to determine if they were correlated using Mantel's test (49). A negative correlation indicates that populations that are spatially close have similar VC population structures. The significance of the correlation was determined as described in Lapointe and Legendre (29).

\section{RESULTS}

VC group diversity. Twelve VC groups were detected among the five populations sampled in 1996, while $29 \mathrm{VC}$ groups were found among seven populations in 2009. A total of $75 \%$ (9 of 12) of the VC groups found in 1996 were detected again in 2009. The VC diversity and structure of $C$. parasitica populations was strongly influenced by the presence of mycovirus. Recovering sites, where mycoviruses are present, averaged 2.7 and 3.7 VC groups per site in 1996 and 2009, respectively. The increase in the number of VC groups at recovering sites in 2009 was due entirely to an increase in the number of VC groups at FR where the number of VC groups increased from four to eight (Figs. 1 and 2). Further, the frequency of the two dominant VC groups at FR changed dramatically between the sampling dates; the most common VC group in 1996 was found at almost half the frequency in 2009 (80\% versus 43\%) and the second most common VC group in 1996 more than doubled in frequency in 2009 (13\% versus $30 \%$ ) (Figs. 1 and 2). CL and RC were both dominated by a single VC group in 1996, which remained dominant in 2009 (Figs. 1 and 2). However, the most striking feature of each recovering population was that all VC groups within a site were unique to that particular site (Figs. 1 and 2) aside from the main group at County Line (MI-5), which was also found at two of the epidemic sites, MD and LE.

In contrast with the pattern in recovering populations, 5 out of the 29 different VC groups $(17 \%)$ were found in multiple epidemic populations. Group MI-2 was detected at all four epidemic sites in 2009, while MI-1 and MI-5 were found at three sites and

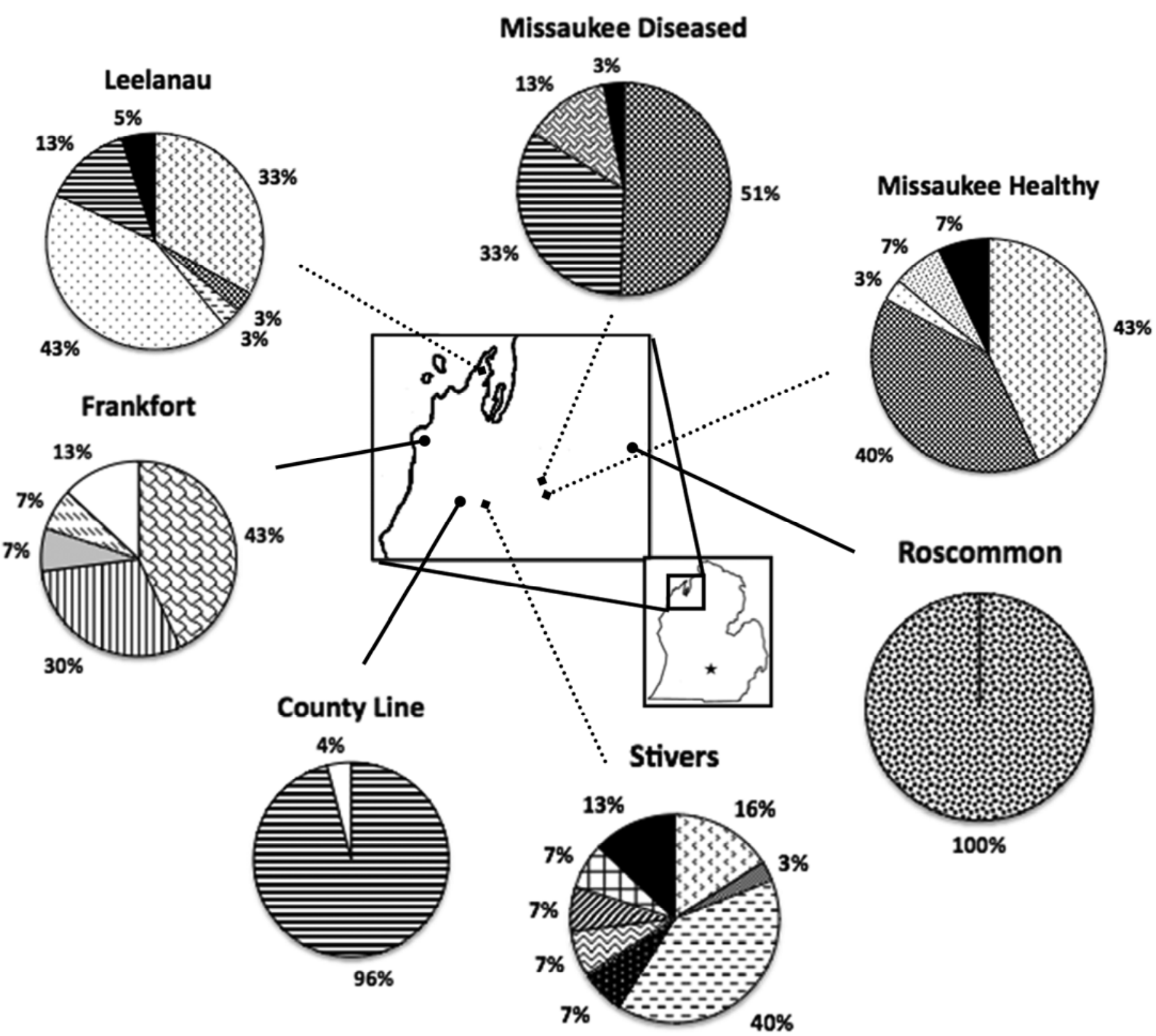

Fig. 2. Vegetative compatibility diversity data at seven Michigan population sites for 2009. Rare types at recovering and epidemic populations are represented by white and black slices, respectively. Recovering sites are denoted with solid circles, epidemic sites with solid squares. 
MI-3 and MI-4 were found at two sites (Figs. 1 and 2). Despite these shared VC groups, the overall pattern of VC diversity was not correlated with physical distance between populations $(r=$ $0.29, P<0.25)$. The lack of correlation is especially curious when considering two epidemic sites, $\mathrm{MH}$ and MD; the sites are separated by less than a kilometer but share only a single VC group, MI-2 (Fig. 2). We have monitored the trees at MD and MH since 1996 (16, A. M. Jarosz and J. C. Springer, unpublished data) and the first infected tree at MH was detected in 1997. In the 15 years since the first infection at $\mathrm{MH}, \mathrm{VC}$ diversity has increased to a level that is comparable with the MD site that has been infected since the late 1980s (D. W. Fulbright, personal observation). Infected trees were also noted in 1997 at LE and by 2009 the number of VC groups and overall VC diversity was similar to that found at MD and $\mathrm{MH}$, sites that are in the main area of Michigan

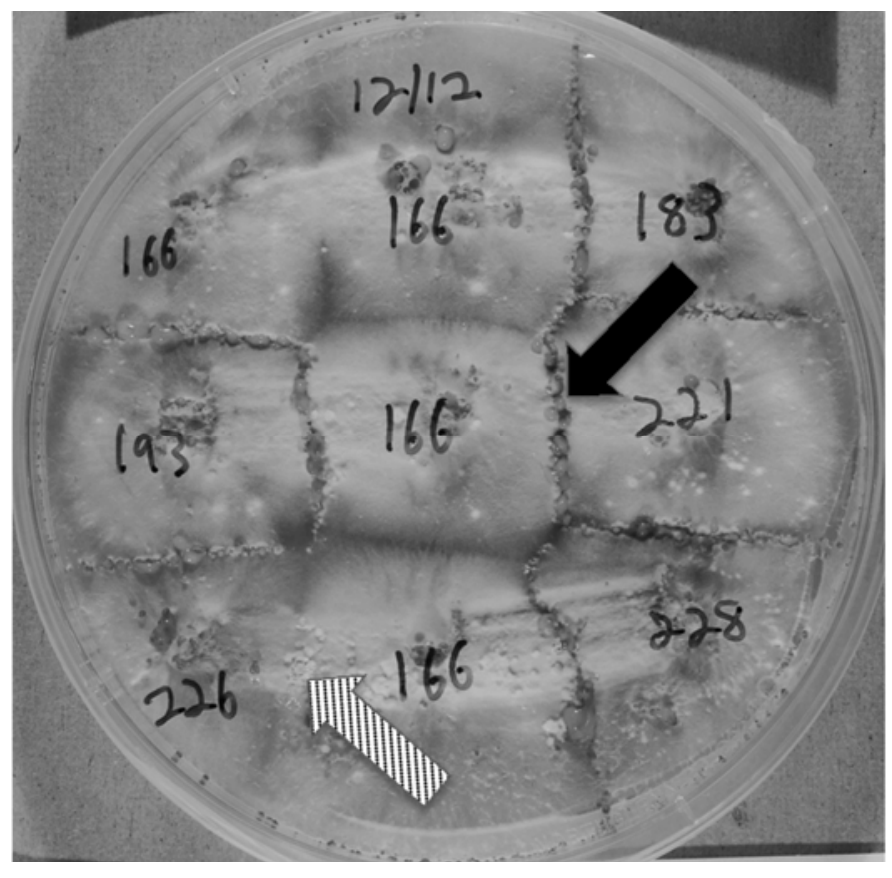

Fig. 3. Vegetative compatibility testing on chestnut stem pieces. Compatible reaction (dashed arrow) between sample 226 and 166, incompatible reaction (solid arrow) between samples 166 and 221. where chestnut blight is found. Generally, epidemic sites had more VC groups than recovering sites, 6.5 in 1996 and 6.75 in 2009 and, although not statistically significant, trends indicated higher overall diversity for VC groups in epidemic sites (Table 1). The number of VC groups increased from 9 to 11 at ST from 1996 to 2009, while the number of VC groups remained constant at MD.

Mating-type allele frequencies. Two recovering populations, $\mathrm{CL}$ and $\mathrm{RC}$, had mating-type allele frequencies that were highly skewed toward the MAT2 allele in both 1996 and 2009 (Table 2). These two populations deviated significantly from the expectation of a 50:50 mating-type ratio that would be expected if these $C$. parasitica populations were regularly going through a sexual cycle. The third recovering population, FR, was skewed toward the MAT2 allele but frequencies were not as extreme as found at $\mathrm{CL}$ and $\mathrm{RC}$, and allele frequencies did not deviate significantly from 50:50 in either 1996 or 2009. As explained below, we suspect that the $C$. parasitica population at FR is gradually escaping mycovirus infection.

The mating-type allele frequencies at the epidemic populations that were infected before 1980 (ST and MD) did not differ significantly from a 50:50 mating-type ratio in either 1996 or 2009. Ratios were closer to 50:50 in 2009 at both sites. Contrary to recovering populations, ST and MD displayed a slight, but persistent skew towards MAT1 at both sampling periods. Mating-type ratios deviated significantly from 50:50 at MH and LE in 2009. Trees at the MH and LE sites were disease-free in 1996 and C. parasitica was first noted at both sites in 1997. Despite the fact that both $\mathrm{MH}$ and LE deviate from a 50:50 mating-type ratio, $\mathrm{MH}$ was skewed toward MAT1, while the $C$. parasitica population at LE was skewed toward MAT2. The excess of MAT1 at MH could possibly be due to its juxtaposition to MD, which is only $300 \mathrm{~m}$ away from $\mathrm{MH}$ and displayed a slight excess of MAT1 in both 1996 and 2009.

\section{DISCUSSION}

Vegetative incompatibility systems in fungi are thought to operate as self/non-self recognition systems that function to thwart the invasion of alien nuclei and mitochondria as well as parasitic elements (e.g., mycoviruses) that inhabit the cytoplasm $(2,6,8,20,22,30,42,46,50)$. To minimize the probability of invasion by alien entities, negative frequency-dependent selection should

TABLE 1. Summary diversity statistics for epidemic and recovering populations

\begin{tabular}{|c|c|c|c|c|}
\hline Site type & Total number of VC groups ${ }^{\mathrm{a}}$ & Simpson \pm 2 SE & Shannon \pm 2 SE & $\begin{array}{c}\text { Maximum Shannon } \\
\text { (\% of maximum) }\end{array}$ \\
\hline Epidemic (LE, MH, ST, MD) & 19 & $5.67 \pm 1.03$ & $1.99 \pm 0.36$ & $2.94(67.6)$ \\
\hline Recovering (RC, CL, FR) & 11 & $3.04 \pm 0.65$ & $1.25 \pm 0.27$ & $2.39(52.1)$ \\
\hline
\end{tabular}

a Vegetative compatibility (VC) richness represents the total time period from 1996 to 2009.

TABLE 2. Distribution of mating types within Cryphonectria parasitica populations in Michigan ${ }^{\mathrm{a}}$

\begin{tabular}{|c|c|c|c|c|c|c|c|c|}
\hline \multirow[b]{2}{*}{ Population } & \multicolumn{4}{|c|}{1996} & \multicolumn{4}{|c|}{2009} \\
\hline & $M A T 1$ & $M A T 2$ & Mixed & $\chi^{2}$ & $M A T 1$ & $M A T 2$ & Mixed & $\chi^{2}$ \\
\hline \multicolumn{9}{|l|}{ Recovering } \\
\hline CL & 2 & 12 & 1 & $7.14 * \mathrm{~b}$ & 3 & 18 & 1 & $10.71 *$ \\
\hline $\mathrm{RC}$ & 4 & 15 & 0 & $6.37 *$ & 5 & 21 & 0 & $9.85 *$ \\
\hline $\mathrm{FR}$ & 9 & 15 & 3 & $1.50 \mathrm{n} . \mathrm{s}$ & 12 & 17 & 3 & 0.86 n.s. \\
\hline \multicolumn{9}{|l|}{ Epidemic } \\
\hline ST & 10 & 5 & 0 & 1.67 n.s. & 15 & 13 & 1 & 0.14 n.s. \\
\hline MD & 11 & 4 & 0 & 3.27 n.s. & 15 & 11 & 7 & 0.62 n.s. \\
\hline MH & $\ldots{ }^{c}$ & $\ldots$ & $\ldots$ & $\ldots$ & 20 & 8 & 1 & $5.14^{*}$ \\
\hline $\mathrm{LE}$ & $\ldots$ & $\ldots$ & $\ldots$ & $\ldots$ & 9 & 20 & 2 & $4.17 *$ \\
\hline
\end{tabular}

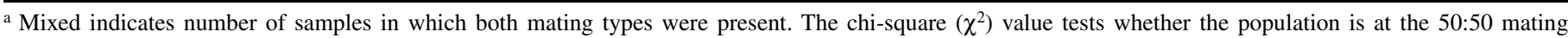
frequency expected if sexual recombination is occurring regularly.

${ }^{\text {b }}$ Critical chi-square for $P=0.05$ is 3.84 , with one degree of freedom $=1$. $*$ indicates significance.

c $\mathrm{MH}$ and LE were blight-free in 1996. 
operate to maximize variability at loci controlling self-incompatibility within fungal populations $(11,38,42)$. If this hypothesis is correct, then we expect the number of VC groups within a population to increase over time and overall VC diversity should also increase. High VC diversity is expected to slow or even stop the spread of mycoviruses within a fungal population.

Patterns of VC diversity in $C$. parasitica have been used to explain the success or failure of mycovirus mediated recovery in European and American chestnut. Mycovirus invasion has been common in Europe where VC diversity is low (39), perhaps due to low diversity among the founders of European populations. For example, VC type EU-1 is found at high frequency in C. parasitica populations on the Black Sea coast of Turkey (1), while EU-2 and EU-5 are common in northern Italy, southern France, Switzerland, and eastern Spain (48). Another VC type, EU-12, is dominant in Bulgaria, Sicily, Romania, southern Italy, and Greece, comprising $82 \%$ of isolates collected while VC type EU-1 is present at low frequency, around $1 \%$. Similar situations can be found elsewhere in Europe where it appears that a few founders at a site results in the proliferation of one to three groups (53). In contrast to the successful spread of mycoviruses in Europe, the phenomenon is rare in North America; the only known natural invasions occurring in Michigan $(19,39,44)$. Several authors have hypothesized that the inability of mycoviruses to gain a foothold in North American populations is due to the relatively high VC diversity $(5,14,26,39,43,47)$. Our results are superficially consistent with this hypothesis. There was a tendency for a greater number of VC groups and higher VC diversity within C. parasitica populations where mycoviruses were absent, although the values were not statistically significant (Table 1). In addition, mating-type allele frequencies suggested that the pathogen populations at the two epidemic populations with a long history of infection (MD and ST) were sexually reproducing. However, if strong negative frequency dependent selection was operating, the number of VC groups and evenness of their distribution should increase over time. This was not the case at the ST and MD epidemic sites where the number of $\mathrm{VC}$ groups increased slightly at ST and not at all at MD (Figs. 1 and 2). Further, VC diversity actually declined at both ST and MD between 1996 and 2009. We suggest an alternate hypothesis in which mycovirus invasion actually leads to a reduction in $C$. parasitica $\mathrm{VC}$ diversity over time. In the absence of mycovirus, $C$. parasitica would be expected to regularly undergo sexual reproduction resulting in recombining of genes controlling VC. However, mycoviruses are known to inhibit sexual reproduction in $C$. parasitica $(3,25,39)$. Work over the past 25 years with mycoviruses found at the CL site and another mycovirus found at Grand Haven, Michigan confirm that the CHV3 mycoviruses do reduce pathogen growth, conidia production, and largely inhibit sexual reproduction (D. W. Fulbright and M. L. Double, personal observation). Further, these CHV3 mycoviruses are transmitted by hyphal anastomosis in a manner similar to that of CHV1 mycoviruses from Europe (28). The persistent and significant skewing of mating-type allele frequencies at CL and RC in 1996 and 2009 also suggest that the C. parasitica populations at these two sites are not reproducing via sexual reproduction (Table 2). This trend is important because selection at these two recovering sites would then operate on asexual populations in which the most fit pathogen multilocus genotype by mycovirus combination would be favored. In consequence, the number of VC groups should decline over time due to the combined processes of selection and genetic drift. We also suggest that the process is reversible and mycoviruses can be lost from $C$. parasitica populations when conditions are not favorable for the maintenance and spread of mycoviruses. We suspect that C. parasitica at FR may be shedding its mycovirus since the percentage of isolates containing mycovirus declined from more than $90 \%$ in 1996 (15) to $76 \%$ in 2009 (J. C. Springer, unpublished data). The decline in mycovirus frequency is paralleled by a doubling in the number of $\mathrm{VC}$ groups from four to eight between 1996 and 2009 and an increase in Shannon diversity (Table 1). Perhaps in consequence, trees at FR have begun to die back over the past 4 years, which contrasts to the gradual size increase occurring at $\mathrm{CL}$ and $\mathrm{RC}$, the other two sites where mycoviruses occur at high frequency (J. C. Springer and A. M. Jarosz, unpublished data).

Results from this study suggest that mycoviruses have a significant influence on the VC structure of $C$. parasitica populations. When mycoviruses attain high frequency, the processes of sexual reproduction and selection in the $C$. parasitica pathogen population may be altered in a manner that leads to reduced VC diversity over time. Selection may act to favor a single most fit mycovirus-fungal genotype combination, while the inhibition of the sexual cycle reduces the formation of new VC groups. Thus, VC diversity within $C$. parasitica populations will be influenced by conditions that favor invasion of mycoviruses into pathogen populations and the concomitant changes in pathogen virulence. What is not known at present is how the underlying vic locus diversity is influenced by mycovirus invasion. We hypothesize that vic loci that have a strong influence on mycovirus transmission will be influenced more than vic loci that have little influence on mycovirus transmission (28). Based on this hypothesis, mycovirus invasion may influence genome-wide diversity within C. parasitica populations at recovering sites in Michigan.

\section{ACKNOWLEDGMENTS}

NSF grants DEB 9623416 and DEB 9509034 and The Hanes Trust of Kalamazoo, Michigan, provided support for this work. We thank the landowners for providing access to their land. Matthew R. Kolp provided technical assistance. This paper was improved by the comments and suggestion of an anonymous reviewer to add the mating-type analysis.

\section{LITERATURE CITED}

1. Akilli, S., Katircioglu, Y. Z., and Maden, S. 2009. Vegetative compatibility types of Cryphonectria parasitica causal agent of chestnut blight, in the Black Sea region of Turkey. For. Pathol. 39:390-396.

2. Anagnostakis, S. L. 1977. Vegetative incompatibility in Endothia parasitica. Exp. Mycol. 1:306-316.

3. Anagnostakis, S. L. 1987. Chestnut blight: The classical problem of an introduced pathogen. Mycologia 79:23-37.

4. Anagnostakis, S. L. 2001. American chestnut sprout survival with biological control of the chestnut-blight fungus population. For. Ecol. Manag. 152:225-233.

5. Anagnostakis, S. L., Hau, B., and Kranz, J. 1986. Diversity of vegetative compatibility groups in Cryphonectria parasitica in Connecticut and Europe. Plant Dis. 70:536-538.

6. Bertrand, H. 2000. Role of mitochondrial DNA in the senescence and hypovirulence of fungi and potential for plant disease control. Annu. Rev. Phytopathol. 38:397-422.

7. Brewer, L. G. 1995. Ecology of survival and recovery from blight in American chestnut trees (Castanea dentata (Marsh.) Borkh.) in Michigan. B. Torrey Bot. Club. 122:40-57.

8. Burdon, J. J., and Thrall, P. H. 2008. Pathogen evolution across the agroecological interface: Implications for disease management. Evol. Appl. 1:57-65.

9. Brasier, C. M. 1995. Episodic selection as a force in fungal microevolution with special reference to clonal speciation and hybrid introgression. Can. J. Bot. 73:S1213-S1221.

10. Brasier, C. M. 2001. Rapid evolution of introduced plant pathogens via interspecific hybridization. Bioscience 51:123-133.

11. Brusini, J., Robin, C., and Franc, A. 2011. Parasitism and maintenance of diversity in a fungal vegetative incompatibility system: The role of selection by deleterious cytoplasmic elements. Ecol. Lett. 14:444-452.

12. Carbone, I., Liu, Y., Hillman, B. I., and Milgroom, M. G. 2004. Recombination and migration of Cryphonectria hypovirus 1 as inferred from gene genealogies and the coalescent. Genetics 166:1611-1629.

13. Colwell, R. K. 2005. EstimateS: Statistical estimation of species richness and shared species from samples. Version 7.5. User's Guide and Application published at http://purl.oclc.org/estimates

14. Cortesi, P., McCulloch, C. E., Song, H. Y., Lin, H. Q., and Milgroom, M. G. 2001. Genetic control of horizontal virus transmission in the chestnut 
blight fungus, Cryphonectria parasitica. Genetics 159:107-118.

15. Davelos, A. L. 1999. Double-stranded RNA mediated recovery of American chestnut populations: A demographic analysis. Ph.D. thesis, Michigan State University, East Lansing, MI.

16. Davelos, A. L., and Jarosz, A. M. 2004. Demography of American chestnut populations: Effects of a pathogen and a hyperparasite. J. Ecol. 92:675-685.

17. Elliston, J. E. 1985. Characteristics of dsRNA-free and dsRNA-containing strains of Endothia parasitica in relation to hypovirulence. Phytopathology 75:151-158.

18. Enebak, S. A., MacDonald, W. L., and Hillman, B. I. 1994. Effect of dsRNA associated with isolates of Cryphonectria parasitica from the central Appalachians and their relatedness to other dsRNAs from North America and Europe. Phytopathology 84:528-534.

19. Fulbright, D. W., Weidlich, W. H., Haufler, K. Z., Thomas, C. S., and Paul, C. P. 1983. Chestnut blight and recovering American chestnut trees in Michigan. Can. J. Bot. 61:3164-3171.

20. Glass, N. L., and Dementhon, K. 2006. Non-self recognition and programmed cell death in filamentous fungi. Curr. Opin. Microbiol. 9:553558.

21. Glass, N. L., Jacobson, D. J., and Shiu, K. T. 2000. The genetics of hyphal fusion and vegetative compatibility in filamentous ascomycetes. Annu. Rev. Genet. 34:165-186.

22. Glass, N. L., and Kaneko, I. 2003. Fatal attraction: Non-self recognition and heterokaryon incompatibility in filamentous fungi. Eukaryot. Cell 2:1-8.

23. Gobbin, D., Hoegger, P. J., Heiniger, U., and Rigling, D. 2003. Sequence variation and evolution of Cryphonectria hypovirus 1 (CHV-1) in Europe. Virus Res. 97:39-46.

24. Grente, J. 1965. Les formes hypovirulentes d'Endothia parasitica et les espoirs de lutte contre le chancre du châtagnier. Academie de l'agriculture de France 1033-1037.

25. Griffin, G. J., and Elkins, J. R. 1986. Chestnut Blight, Other Endothia Diseases, and the Genus Endothia. American Phytopathological Society, St. Paul, MN.

26. Heiniger, U., and Rigling, D. 1994. Biological control of chestnut blight in Europe. Annu. Rev. Phytopathol. 32:581-599.

27. Hill, T. C. J., Walsh, K. A., Harris, J. A., and Moffett, B. F. 2003. Using ecological diversity measures with bacterial communities. FEMS Microbiol. Ecol. 43:1-11.

28. Huber, D. H. 1996. Genetic analysis of vegetative incompatibility polymorphisms and horizontal transmission in the chestnut blight fungus, Cryphonectria parasitica. Ph.D. thesis. Michigan State University, East Lansing, MI.

29. Lapointe, F. J., and Legendre, P. 1992. Statistical significance of the matrix correlation coefficient for comparing independent phylogenetic trees. Syst. Biol. 41:378-384.

30. Lawrence, G. J., Boelen, M. G., and Pryor, A. 1988. Transmission of double-stranded RNAs in flax rust, Melampsora lini. Can. J. Bot. 66:6166.

31. Liu, Y. C., and Milgroom, M. G. 1996. Correlation between hypovirus transmission and the number of vegetative incompatibility (vic) genes different among isolates from a natural population of Cryphonectria parasitica. Phytopathology 86:79-86.

32. MacDonald, W. L., and Fulbright, D. W. 1991. Biological control of chestnut blight: Use and limitations of transmissible hypovirulence. Plant Dis. 75:656-661.

33. Magurran, A. 1988. Ecological Diversity and Its Measurement. Princeton University Press, Princeton, NJ.

34. Marra, R. E., and Milgroom, M. G. 2001. The mating system of the fungus Cryphonectria parasitica: Selfing and self-incompatibility. Heredity 86:134-143.
35. McCormick, J. F., and Platt, R. B. 1980. Recovery of an Appalachian forest following the chestnut blight or Catherine Keever-you were right! Am. Midl. Nat. 104:264-273.

36. Merkel, H. W. 1906. A deadly fungus on the American chestnut. 10th Annual Report of the New York Zoological Society. The Crow Press, New York.

37. Micali, C. O., and Smith, M. L. 2006. A nonself recognition gene complex in Neurospora crassa. Genetics 173:1991-2004.

38. Milgroom, M. G., and Cortesi, P. 1999. Analysis of population structure of the chestnut blight fungus based on vegetative incompatibility genotypes. Proc. Natl. Acad. Sci. USA 96:10518-10523.

39. Milgroom, M. G., and Cortesi, P. 2004. Biological control of chestnut blight with hypovirulence: A critical analysis. Annu. Rev. Phytopathol. 42:311-338

40. Mir-Rashed, N., Jacobson, D. J., Dehghany, M. R., Micali, O. C., and Smith, M. L. 2000. Molecular and functional analyses of incompatibility genes at het-6 in a population of Neurospora crassa. Fungal Genet. Biol. 30:197-205.

41. Morozov, A. Y., Robin, C., and Franc, A. 2007. A simple model for the dynamics of a host-parasite-hyperparasite interaction. J. Theor. Biol. 249:246-253.

42. Nauta, M. J., and Hoekstra, R. F. 1994. Evolution of vegetative incompatibility in filamentous ascomycetes. I. Deterministic models. Evolution 48:979-995

43. Nuss, D. L. 1992. Biological control of chestnut blight: An example of virus-mediated attenuation of fungal pathogenesis. Biol. Control 56:561576.

44. Peever, T. L., Liu, Y. C., and Milgroom, M. G. 1997. Diversity of hypoviruses and other double-stranded RNAs in Cryphonectria parasitica in North America. Phytopathology 87:1026-1033

45. Potter, C., Harwood, T., Knight, J., and Tomlinson, I. 2011. Learning from history, predicting the future: The UK Dutch elm disease outbreak in relation to contemporary tree disease threats. Phil. Trans. R. Soc. B. 366:1966-1974.

46. Rayner, A. D. M. 1991. The phytopathological significance of mycelial individualism. Annu. Rev. Phytopathol. 29:305-323.

47. Robin, C., Anziani, C., and Cortesi, P. 2000. Relationship between biological control, incidence of hypovirulence and diversity of vegetative compatibility types of Cryphonectria parasitica in France. Phytopathology 90:730-737.

48. Robin, C., and Heiniger, U. 2001. Chestnut blight in Europe: Diversity of Cryphonectria parasitica, hypovirulence and biocontrol. For. Snow Landsc. Res. 367:361-367.

49. Rohlf, F. J. 1998. NTSYSpc. Numerical taxonomy and multivariate analysis system. Version 2. User Guide. Applied Biostatistics, Inc., Setauket, NY.

50. Roper, M., Ellison, C., Taylor, J. W., and Glass, N. L. 2011. Nuclear and genome dynamics in multinucleate ascomycete fungi. Curr. Biol. 21:R786-R793.

51. Russin, J. S., and Shain, L. 1985. Disseminative fitness of Endothia parasitica containing different agents for cytoplasmic hypovirulence. Can. J. Bot. 63:54-57.

52. Santini, A., Pecori, F., Pepori, A., and Brookes, A. 2012. Morfeo elm: A new variety resistant to Dutch elm disease. For. Pathol. 42:171-176.

53. Sotirovski, K., Milgroom, M. G., Rigling, D., and Heiniger, U. 2006. Occurrence of Cryphonectria hypovirus 1 in the chestnut blight fungus in Macedonia. For. Pathol. 36:136-143.

54. Taylor, D. R., Jarosz, A. M., Lenski, R. E., and Fulbright, D. W. 1998. The acquisition of hypovirulence in host-pathogen systems with three trophic levels. Am. Nat. 151:343-355.

55. Wu, J., Saupe, S. J., and Glass, N. L. 1998. Evidence for balancing selection operating at the het-c heterokaryon incompatibility locus in a group of filamentous fungi. Proc. Natl. Acad. Sci. USA 95:12398-12403. 